\title{
Relative Ratio of Mature Pustules: A Simple Method to Assess Partial Resistance of Barley to Puccinia hordei
}

Guo-Liang Jiang, State Key Laboratory of Crop Genetics and Germplasm Enhancement, Nanjing Agricultural University (NAU), Nanjing 210095, P.R. China; Thierry C. Marcel, Laboratory of Plant Breeding, Graduate School of Experimental Plant Sciences, Wageningen University, P.O. Box 386, NL-6700 AJ Wageningen, the Netherlands; Fernando Martínez, Instituto de Agricultura Sostenible, C.S.I.C., Apdo. 4084, E-14080 Córdoba, Spain; and Rients E. Niks, Laboratory of Plant Breeding, Graduate School of Experimental Plant Sciences, Wageningen University (WUR), P.O. Box 386, NL-6700 AJ Wageningen, the Netherlands

\begin{abstract}
Jiang, G.-L., Marcel, T. C., Martínez, F., and Niks, R. E. 2007. Relative ratio of mature pustules: A simple method to assess partial resistance of barley to Puccinia hordei. Plant Dis. 91:301-307.

In plant breeding and germplasm evaluation, large-scale assessment of quantitative resistance is desirable, but feasible only if a simple and accurate measure is available. In several plantpathogen systems, latent period (LP) is a parameter that is well correlated with the level of partial resistance observed in field trials. However, measuring LP or relative LP (RLP), i.e., relative to the reference accessions, is laborious. We investigated the value of relative ratio of mature pustules (RRMP, relative to the susceptible control) as a simple and rapid alternative to replace LP estimation in barley to barley leaf rust (Puccinia hordei). A set of $103 \mathrm{~F}_{9}$ recombinant inbred lines (RILs) derived from a cross L94 $\times$ Vada was sown in a greenhouse compartment, and was inoculated at the seedling stage with isolate Uppsala or 24, and at the adult plant stage with isolate 24. In demarcated sections of leaves, the number of mature pustules was counted several times after inoculation. The ratio of mature pustules (RMP) and LP50 were calculated to assess RRMP and RLP, respectively, and to identify the quantitative trait loci (QTLs) contributing to the genetic variation. The contrasts in RRMP among accessions were highest when the susceptible reference line had developed 70 to $90 \%$ mature orange pustules, the immature infection sites being visible as pale flecks. At this optimal time of observation, the correlation between RRMP and RLP in both the seedling stage and the adult plant stage was highly significant $(r=-0.82 \sim$ -0.98). Compared with RLP, RRMP was much easier and simpler to measure and still showed good correspondence with RLP in the identification of QTLs for partial resistance. In another experiment at the seedling stage with 25 barley cultivars and lines inoculated with isolate 1.2.1, the coefficient of correlation between RRMP and RLP was -0.98 . Therefore, we conclude that RRMP should have great application potential in breeding programs and germplasm screening and could be used in fundamental studies as well.
\end{abstract}

In many plant pathosystems there are two distinct forms of resistance: qualitative, race-specific resistance, and quantitative, race-non-specific resistance $(19,21,22)$. In general, qualitative resistance inherits monogenically or oligogenically and is

Corresponding authors:

G.-L. Jiang, E-mail: gljiang@msu.edu or gljiang99@yahoo.com

R. E. Niks, E-mail: Rients.Niks@wur.nl

Current address of G.-L. Jiang: Department of Crop and Soil Sciences, Michigan State University, East Lansing 48824.

Current address of F. Martínez: EUITA (Universidad de Sevilla) Ctra. Utrera Km.1 41013 Sevilla Spain.

This research was made possible by a visiting scholarship in the framework of WUR-NAU collaboration, Wageningen, the Netherlands.

Accepted for publication 3 October 2006.

DOI: 10.1094/PDIS-91-3-0301

(C) 2007 The American Phytopathological Society ephemeral. This resistance is almost always based on a hypersensitive reaction of plant tissue to the presence of the pathogen. In contrast, quantitative resistance is polygenic, not based on a hypersensitive reaction, and presumably more durable (13). During the early history of plant breeding for disease resistance, the emphasis was put on qualitative resistance because of the simplicity of assessment and the usually complete protection provided by the resistance allele. However, frequent loss of effectiveness of this resistance taught plant breeders that quantitative, race-non-specific resistance deserves more attention. Consequently, polygenic resistance has been increasingly investigated in field crops since the 1970s (7).

A simple and accurate method to measure quantitative resistance is critical for large-scale screening of germplasm. In field trials, the severity of infection can be estimated and converted to the area under the disease progress curve (AUDPC). In greenhouse trials, a monocyclic evaluation can be performed. In some plant pathosys- tems, the latent period (LP) of the pathogen at the adult plant stage in the greenhouse is well correlated with the AUDPC measured in the field $(1,14,17)$. LP and AUDPC have been widely used to evaluate partial resistance in different plantpathogen systems, such as wheat-Puccinia recondita (15), pearl millet-Puccinia substriata (24), tropical pasture legume Stylosanthes hamata-Colletotrichum gloeosporioides (6), potato-Phytophthora infestans (3), and poplar-Melampsora larici-populina (4). Currently, LP and AUDPC are the major methods to assess partial resistance in barley to leaf rust, especially in fundamental studies $(2,12,17)$. In breeding programs, resistance is usually determined in the field by estimating the severity of disease in a 1 to 9 scale or similar coding method. However, such a measurement is less accurate and more subjective. Measuring LP or AUDPC requires several disease assessments or observations for each accession or single plant. It is too laborious to assess these parameters on a large scale in plant breeding. A simpler measure, which can be easily implemented for the assessment of partial resistance on a large scale, would be of great use for plant breeding and research. The objectives of our study were to: (i) evaluate a simple parameter for the assessment of pathogen development rate that could replace measurement of LP: the relative ratio of mature pustules; and (ii) provide advice on how to employ this measure in plant breeding and germplasm screening programs, particularly for use in assessments conducted with controlled inoculations under greenhouse conditions.

\section{MATERIALS AND METHODS}

Experiment I. Host and pathogen. A set of $103 \mathrm{~F}_{9}$ recombinant inbred lines (RILs) derived from a cross of barley (Hordeum vulgare L.) cultivars L94 $\times$ Vada was sown in the greenhouse at Wageningen University and used as the host plants. The parent L94 is extremely susceptible, and Vada has a high level of partial resistance to barley leaf rust (Puccinia hordei) $(14,17)$. Barley leaf rust fungal isolate Uppsala or 24 was used as the pathogen for seedling stage 
experiments, and isolate 24 for adult plant stage experiment.

Seedling stage experiments. For evaluation at the seedling stage, the seeds of the 103 RILs and two parents were sown in 37 $\times 39 \mathrm{~cm}$ wood trays filled with soil Lentse potgrond $\mathrm{nr} 4$ (15\% loam, 20\% sphagnum, and $65 \%$ peat) in the greenhouse. In the first experiment, a total of 18 trays were planted. In each tray, six RILs and two parents (as controls) were planted in two rows parallel to the longer sides of the tray, with three RILs and a control in each row. The RILs were randomly assigned to the trays. For each RIL and control, six to eight seeds were sown in succession and five to six seedlings retained. The planting of all 103 RILs was accomplished on three different dates at an interval of 8 days, with six trays planted for each planting date. About 10 days after sowing (Zadoks growth stage 11), the first leaves were fixed horizontally onto the soil with the adaxial surface upward, by pinning the leaves down with $U$ pins. Inoculation with isolate 24 was conducted in an inoculation tower, by dusting a mixture of fresh urediospores and lycopodium powder $(1: 10)$ on the seedlings. Three milligrams of spores were applied per tray, which resulted in 100 to 150 urediospores per $\mathrm{cm}^{2}$ leaf area. After inoculation, the trays were moved to a greenhouse compartment equipped with a humidifier. Inoculated plants were incubated overnight (8:00 P.M. through 6:00 A.M.) in the dark at 100\% relative humidity and about $18^{\circ} \mathrm{C}$. Next morning, the trays were transferred to a greenhouse compartment where the temperature was set to oscillate over 20 to $22^{\circ} \mathrm{C}$. Since the experimental conditions (soil, temperature, humidity, inoculum, etc.) were well controlled and highly uniform among planting dates or trays, the experiment was analyzed as a completely randomized design (CRD) with individual seedlings as replications. In the second experiment, a total of 12 trays were planted. In each tray, 36 RILs and two parents were planted in two rows each having 18 RILs plus two parents (as controls). The RILs were randomly assigned to the trays and rows, with one seedling per line per row. Each RIL was replicated four times, i.e., each RIL had four seedlings randomly allocated within the experiment. The planting was done on two dates with six trays planted for each planting date. All the 103 seedlings of RILs plus six seedlings of each parent planted on the same day and on the same side of the six trays constituted a replication. For each parent, however, the six seedlings from the same side of the six trays (planted on the same day) were pooled for analysis since the differences among the six seedlings within such a replication were not significant $(P>0.35)$; in other words, each of the two parents had four replications with each replication having six seedlings.
At Zadoks growth stage 11, inoculation with isolate Uppsala was conducted as described above. Similar to the first experiment, the experimental conditions were highly uniform, and thus the data were analyzed as CRD.

When the pale flecks of immature pustules appeared, an area that contained 50 to 60 flecks was demarcated on each leaf. Orange-brown mature pustules within the marked area were counted once daily from the sixth day after incubation (the overnight moist period) until the number did not increase within 2 days, but twice a day on the sixth and seventh day in the first experiment. The latent period (LP50) was calculated as the number of hours from midnight after inoculation until the time that $50 \%$ of the ultimate number of pustules had matured based on orange-brown color or sporulation.

$\mathrm{LP50}=T_{1}+\left[\left(T_{2}-T_{1}\right) \times\left(N_{50}-N_{1}\right) /\left(N_{2}-N_{1}\right)\right]$

$T_{1}$ and $T_{2}$ are the times (in hours) elapsed from midnight after inoculation to the last counting before $N_{50}$ (half the final number of mature pustules) and the first counting after $N_{50}$, respectively; $N_{1}$ and $N_{2}$ represent the numbers of sporulating pustules at $T_{1}$ and $T_{2}$.

For each tray in the first experiment, the average of five seedlings of L94 was calculated and used to calculate the relative latent period (RLP) of the RILs in the same tray (12). For each replication of the second experiment, the average LP50 of six seedlings of L94 was calculated. This value was adjusted to 100 and used to calculate the RLP of the RILs in the same replication (12).

The ratio of mature pustules (RMP) for each evaluation time was calculated as the ratio of the number of mature pustules at time $T_{x}$ to the final number of pustules in the demarcated area. For each tray in the first experiment or each replication in the second experiment, the mean RMP of L94 was set at 100 to calculate the relative ratio of mature pustules (RRMP) for the RILs.

Adult plant stage experiment. For adult plant evaluations, a randomized design was adopted with three replications. The experiment was conducted in a greenhouse compartment. For each RIL, three seedlings were grown per $11 \times 11 \mathrm{~cm}$ pot or replication. The parents (L94 and Vada) were sown as references many times at intervals of 1 week so that they could be included in each inoculation. Because of the large number of RILs and their differences in development rate, the plants were grouped when flag leaves fully extended from the sheaths and inoculated at Zadoks growth stage 40-49. For each inoculation, two pots for each of L94 and Vada were included as references. Inoculation with isolate 24 was conducted by dusting a mixture of fresh urediospores and lycopodium powder $(1: 10)$ on the leaves with a powder blower. Afterward, the pots were placed in the incubation room and incubated overnight at $100 \%$ relative humidity in the dark. Next day, the pots were transferred to another greenhouse compartment with ambient temperature $\left(20\right.$ to $\left.24^{\circ} \mathrm{C}\right)$ and 70 to $90 \%$ relative humidity. Mature pustules in a demarcated section of the flag leaves were counted once a day, beginning 6 days after inoculation and ending when the pustule number did not increase over 2 days. For each RIL, three to six flag leaves were observed per pot. The LP50, RLP, RMP, and RRMP were computed as described for seedling tests.

Statistical analysis and QTL mapping. A one-way ANOVA for RLP and RRMP was conducted as a completely randomized design analysis (20). For seedling stage, since only one leaf was sampled per plant, the data from individual leaves were used for the analysis. For adult plant stage, the averages of all the sampled leaves within a replication (or pot) were used for the analysis. Broad-sense heritability on an RIL mean basis was estimated based on the results of ANOVA using the formula $h_{b}{ }^{2}=\sigma_{g}{ }^{2} /\left(\sigma_{g}{ }^{2}+\sigma_{e}{ }^{2} / r\right)(5)$, i.e., the expected mean square of RILs was used to estimate the genotypic variance, and the mean square of the error was used to estimate the environmental variance, respectively. Correlation between RRMP and RLP at the different evaluation times was also calculated at each growth stage.

QTL mapping and identification of the molecular markers associated with the partial resistance were performed as described by Qi et al. (16). A dense linkage map covering the barley genome (1062 $\mathrm{cM})$ and containing 566 AFLP markers was constructed by Qi and colleagues (18). A skeletal map with a uniform distribution of markers with about 5-cM intervals was extracted and used for mapping QTLs $(16,17)$. A computer program, MapQTL version 5.0 (23), was used for interval mapping (10) and multiple-QTL mapping (MQM) (8) to identify QTLs for the RLP and RRMP. Interval mapping was initially used to detect the region of putative QTLs. The marker with the highest logarithm of odds (LOD) value was then taken as a cofactor for running the MQM program. This was repeated until a stable LOD profile was reached. Then the same set of cofactors was used to run restricted multipleQTL mapping (rMQM). An LOD value of 3.0 was set as the threshold value for declaring a QTL. The results obtained with the $\mathrm{rMQM}$ method are presented in this paper. For a QTL whose LOD value was lower than 3.0 for rMQM but significant for MQM, the data are also presented and declared a valid QTL.

Experiment II. In order to further validate the effectiveness of RRMP in evaluation of the level of partial resistance in plants, 25 barley lines and cultivars that represented different levels of partial resistance in cultivated barley to $P$. hordei were 
inoculated in the seedling stage. The plants were grown in wooden trays in the greenhouse. Eight lines, five seedlings per line, and the control L94 were sown in each tray. Approximately 11 days after sowing (Zadoks growth stage 11), the seedlings were inoculated with about 180 spores $/ \mathrm{cm}^{2}$ of $P$. hordei isolate 1.2.1. Methods and measuring LP50 were as described above for the seedling tests of the 103 RILs in experiment I. RMP was calculated as the quotient of mature pustules at the first and second counts. The first evaluation time was when, on line L94, 70\% of the pustules had matured and the rest were visible as pale flecks. The second evaluation time was when all the pustules had matured in all the lines. The experiment was conducted four times. The RLP and RRMP were calculated based on L94 as described in experiment I. The average of five leaves of each line was calculated for each replication. One-way ANOVA based on replication means and estimation of broad-sense heritability on a line mean basis were conducted $(5,20)$.

\section{RESULTS}

Experiment I. Ratio of mature pustules in L94 and Vada and the optimal time for measuring RMP. Both the LP50 and the ratio of mature pustules (RMP) obviously differed between the parents L94 and Vada (Fig. 1), reflecting the different levels of partial resistance to barley leaf rust. The difference in RMP on L94 and Vada varied in the course of observations. The largest difference in the RMP between L94 (susceptible) and Vada (partially resistant) was observed when the ratio of mature pustules in L94 was between 70 and $90 \%$ at the seedling stage, and between 75 and $95 \%$ at the adult plant stage, respectively. Under the conditions of this study, the corresponding time for measurement of RMP was from 6.5 to 7.5 days postinoculation (dpi) for the isolate Uppsala and from 6.2 to $6.6 \mathrm{dpi}$ for the isolate 24 in seedlings, and from 7.5 to $9.5 \mathrm{dpi}$ for the isolate 24 in adult plants, respectively (Fig. 1).

Partial resistance to leaf rust in the RILs of L94 $\times$ Vada barley population. ANOVA showed that for RLP and RRMP, at all the evaluation times, RILs were a significant source of variation $(F>2.0, P<0.01)$ (Table 1), but there were overlapping ranges of significant differences among the 103 RILs. Compared to RLP, RRMP had larger coefficients of variation (CV), smaller $F$ values, and lower estimates of heritability at most evaluation times (Table 1). For RRMP, all statistic parameters varied across the times of evaluation. For the seedling stage, the highest $F$ value and estimate of heritability for RRMP was reached at 7.5 dpi with isolate Uppsala and at 6.4 dpi with isolate 24 , and surpassed those for RLP. For the adult plant stage with isolate 24 , the highest $F$ value and estimate of heritability for
RRMP was on 8.5 dpi, which were close to those for RLP.

Frequency distributions of RILs were continuous for both RLP and RRMP (Fig. 2). The RLP distributions appeared to be similar to a normal distribution. The shape of the frequency distribution for RRMP differed among observation times. The RRMP distributions were roughly normal for 6.5 and $7.5 \mathrm{dpi}$ in the seedling stage with isolate Uppsala and 6.4 dpi with isolate 24 , and from 7.5 to $9.5 \mathrm{dpi}$ in the adult plant stage.

Correlation between RLP and RRMP. There was a highly significant correlation between RRMP and RLP at both the seedling stage and the adult plant stage (Table 2). The correlation coefficients varied with the time of observation. For the seedling stage, the correlation coefficient was highest at $7.5 \mathrm{dpi}$ with isolate Uppsala and at 6.7 dpi with isolate 24 . For the adult plant stage with isolate 24 , the highest correlation coefficient was obtained at 8.5 dpi.

The average coefficients of correlation among replications for RRMP (data not shown) were highest at $7.5 \mathrm{dpi}$ with isolate Uppsala (0.71) and at $6.4 \mathrm{dpi}$ with isolate $24(0.78)$ in the seedling stage, respectively, and at $8.5 \mathrm{dpi}$ in the adult plant stage with isolate $24(0.68)$. For RLP, the average coefficients of correlation among replications were 0.70 and 0.69 for isolate Uppsala and 24 in the seedling stage, respectively, and 0.71 for the adult plant stage with isolate 24 . At these evaluation
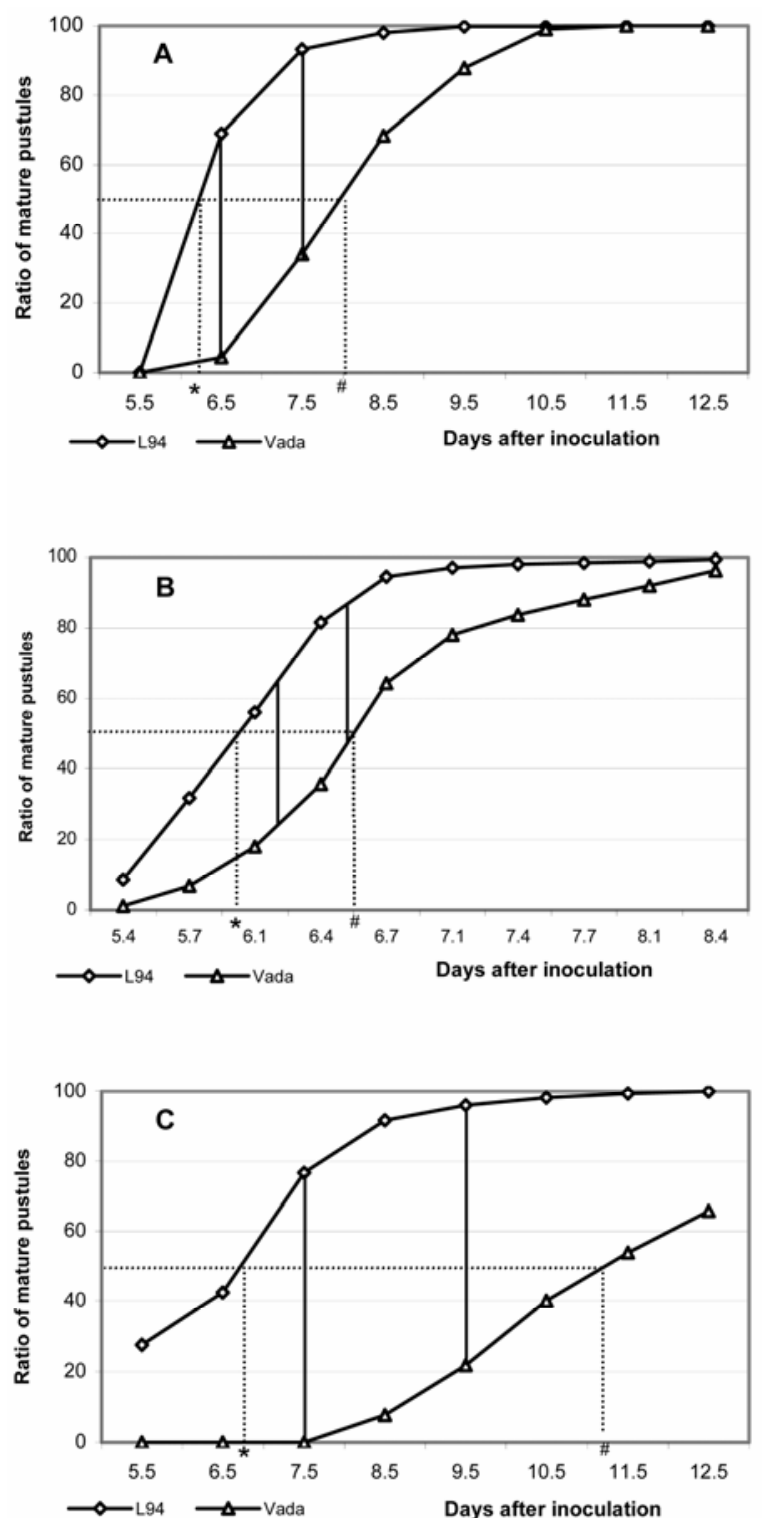

Fig. 1. The ratio of mature pustules (RMP) (\%) for barley leaf rust in L94 and Vada at the seedling stage with $\mathbf{A}$, isolate Uppsala (4 replications each having 6 leaves), and $\mathbf{B}$, isolate 24 (18 replications each having 5 leaves), and $\mathbf{C}$, at the adult plant stage with isolate 24 (10 replications each having 3 to 5 leaves). Indicated are the times that $50 \%$ of the pustules on L94 (*) and on Vada (\#) were mature, marking the LP50 on those accessions. The two vertical solid lines in each of the three graphs indicate the two time points and the corresponding RMP values of L94 when the difference in the RMP between L94 and Vada was greatest. 
times (7.5 dpi for isolate Uppsala and 6.4 dpi for isolate 24 in the seedling stage, and $8.5 \mathrm{dpi}$ for isolate 24 in the adult plant stage), RRMP showed a similar correlation among replications compared to RLP.

In addition, the coefficients of correlation between seedlings and adult plants with isolate 24 were $0.51(P<0.01)$ for RLP and $0.52(P<0.01)$ for RRMP, respectively. The correlation coefficient between seedlings with isolate Uppsala and adult plants with isolate 24 was $0.59(P<$ 0.01 ) for both RLP and RRMP. This suggests that the resistance performance of adult plants could be predicted by seedling resistance to only a moderate extent.

QTLs and molecular markers for partial resistance. The RLP and RRMP of the RILs were mapped on a skeleton map extracted from a high-density AFLP map (18). For seedlings inoculated with isolate Uppsala, four QTLs contributed to the variation in RLP and RRMP (Table 3). Both measures shared the same peak markers for each QTL. There was a good agreement between RLP and RRMP in LOD score and percentage of explained variation for the QTLs. For seedlings inoculated with isolate 24, four QTLs were detected for both RLP and RRMP, although the LOD values of Rphq7 and Rphq3a did not reach the threshold for RRMP. The LOD value and percentage of explained variation of $R p h q 2$ for RLP were distinctly larger than those for RRMP (Table 3). For the partial resistance in adult plants inoculated with isolate 24 , seven QTLs were identified (Table 3). The major QTLs Rphq3, Rphq2, and Rphq4 had higher LOD scores and percentages of explained variation for RRMP than for RLP. In contrast, the values of LOD and explained variation for $R p h q 8$ for RRMP were lower than those for RLP. For Rphq9, RRMP showed a higher LOD value but lower percentage of explained variation than RLP. Rphq10 had significant LOD for RLP, but not for RRMP. In addition, Rphq6 was significant for multiple-QTL mapping (MQM) for RRMP, but not for RLP.

Experiment II. In this experiment with 25 barley lines and isolate 1.2.1 of $P$. hor$d e i$, ANOVA showed that there were highly significant differences among the cultivars or lines in both RLP and RRMP (Table 4). The estimate of broad-sense heritability for RLP was larger than that of RRMP. The correlation between RRMP and RLP was very high $(r=-0.98, P<0.01)$. The average coefficients of correlation among replications were 0.86 for RLP and 0.80 for RRMP, respectively.

\section{DISCUSSION}

Although it would be very useful to develop a simplified alternative screening method for quantitative resistance, few attempts have been reported $(2,9)$. Newton (11) used the ratio of mature lesions (RML) to test the partial resistance of barley to mildew, which was the number of sporulating lesions scored when the first colonies became visible relative to the final number of sporulating lesions or the maximum number of colonies counted. In our study, a similar simplified measure, the relative ratio of mature pustules (RRMP), was used to evaluate the partial resistance to leaf rust in barley. RRMP was computed as the ratio of mature pustules relative to

Table 1. Averages and coefficients of variation (CV) of relative latent period (RLP) and relative ratio of mature pustules (RRMP) of leaf rust at different times (days after inoculation) in a barley recombinant inbred line (RIL) population

\begin{tabular}{|c|c|c|c|c|c|c|c|c|}
\hline & \multirow[b]{2}{*}{ RLP } & \multicolumn{7}{|c|}{ RRMP } \\
\hline & & $6.5 \mathrm{~d}^{\mathrm{a}}$ & $7.5 \mathrm{~d}$ & $8.5 \mathrm{~d}$ & $9.5 \mathrm{~d}$ & & & \\
\hline \multicolumn{9}{|c|}{ Seedling stage/Uppsala ${ }^{\mathrm{b}}$} \\
\hline Average & 113.5 & 37.4 & 69.3 & 87.2 & 95.7 & & & \\
\hline $\mathrm{CV} \%$ & 5.9 & 61.0 & 26.0 & 11.2 & 4.1 & & & \\
\hline$F$ value $^{\mathrm{c}}$ & $8.8^{* *}$ & $6.8 * *$ & $9.3 * *$ & $3.9 * *$ & $2.2^{* *}$ & & & \\
\hline \multirow[t]{2}{*}{$h_{b}^{2} \% \mathrm{~d}^{\mathrm{d}}$} & 88.6 & 85.4 & 89.3 & 74.3 & 54.0 & & & \\
\hline & & $5.7 \mathrm{~d}$ & $6.4 \mathrm{~d}$ & $6.7 \mathrm{~d}$ & & & & \\
\hline \multicolumn{9}{|c|}{ Seedling stage $/ 24^{\mathrm{e}}$} \\
\hline Average & 107.2 & 36.8 & 58.8 & 77.1 & & & & \\
\hline $\mathrm{CV} \%$ & 4.0 & 78.3 & 40.3 & 19.8 & & & & \\
\hline$F$ value & $11.6 * *$ & $12.2 * *$ & $17.6^{* *}$ & $10.1 * *$ & & & & \\
\hline \multirow[t]{2}{*}{$h_{b}^{2} \%$} & 91.4 & 91.8 & 94.3 & 90.1 & & & & \\
\hline & & $6.5 \mathrm{~d}$ & $7.5 \mathrm{~d}$ & $8.5 \mathrm{~d}$ & $9.5 \mathrm{~d}$ & $10.5 \mathrm{~d}$ & $11.5 \mathrm{~d}$ & $12.5 \mathrm{~d}$ \\
\hline \multicolumn{9}{|c|}{ Adult plant stage $/ 24^{\mathrm{f}}$} \\
\hline Average & 130.0 & 21.4 & 38.1 & 55.1 & 72.8 & 83.2 & 89.5 & 94.2 \\
\hline $\mathrm{CV} \%$ & 14.3 & 121.1 & 69.0 & 46.1 & 31.4 & 20.1 & 13.8 & 9.8 \\
\hline$F$ value & $7.2 * *$ & $4.5 * *$ & $5.6^{* *}$ & $6.5^{* *}$ & $6.4 * *$ & $5.1 * *$ & $4.6 * *$ & $4.0 * *$ \\
\hline$h_{b}^{2} \%$ & 86.0 & 77.9 & 82.7 & 84.6 & 84.4 & 80.2 & 78.2 & 74.7 \\
\hline
\end{tabular}

a Days postinoculation.

${ }^{\mathrm{b}}$ Based on four-replication data with isolate Uppsala.

${ }^{c} F=\mathrm{MS}_{\mathrm{RIL}} / \mathrm{MS}_{\mathrm{e}}$ in a one-way ANOVA. $* * P<0.01$.

${ }^{\mathrm{d}} h_{b}{ }^{2}=$ broad-sense heritability (genetic variance divided by phenotypic variance) based on ANOVA.

e Based on five-replication data with isolate 24.

${ }^{\mathrm{f}}$ Based on three-replication data with isolate 24.

the ratio in the susceptible control L94. Our results showed that RRMP was highly correlated to RLP, a more laborious measure that has been used in several plantpathogen systems. Moreover, RRMP also showed a very high similarity or consistency with RLP in the identification of QTLs for partial resistance. Therefore, we conclude that RRMP is a good alternative to RLP, since it is very highly correlated and about as powerful as RLP to identify QTLs for partial resistance.

Compared to RLP, determining RRMP requires less time. Observations for RLP in this experiment were usually conducted six to eight times at both the seedling stage and adult plant stage. In general, pustules need to be counted at least four times, even by very experienced assessors. However, two observations are sufficient to determine RRMP. Actually, it may be possible that only a single counting, when the susceptible line is approaching $90 \%$ mature pustules, would be sufficient. A conservative estimate suggests that RRMP might result in a saving of at least $50 \%$ labor and/or time compared with RLP measurement. If five seedlings or three to four flag leaves at adult plant stage are observed, it will take 2 to $3 \mathrm{~min}$ to collect pustule data per line, thus approximately 25 lines can be assessed in an hour. RRMP could be easily used in breeding programs and germplasm screening on a large scale.

One problem in using RRMP in screening is that it revealed a lower heritability than RLP, except at a few assessment times for plants in the seedling stage. This is inconsistent with the results on RML reported by Niks and Fernández (data published in literature citation 2). In their observation, the heritability of RML (similar to RRMP described here) and RLP (or LP50) were 87 and 76\%, respectively. Our results also showed that the differences in heritability between RRMP at optimal evaluation times and RLP were not big (just 1.4 to $3.3 \%$ ).

RRMP might be more variable than RLP in size of contrast among the accessions in the test, depending on the first observation time, which is very critical for a reliable evaluation of resistance. Counting too early or too late in development of pustules would result in less accurate measures. If counting is done relatively early, differences among the most resistant lines will be small, since the RMP for all of them would be close to zero, but the differences among the most susceptible lines will be more distinct. Alternatively, a relatively late time of first counting will lead to small differences among the most susceptible lines since their RMP would all approach $100 \%$. Therefore, in a population where the range in resistance levels is very wide, RRMP will not allow precise distinction among lines or individuals that are at one extreme.

This study revealed that the optimal assessment time could be determined based 

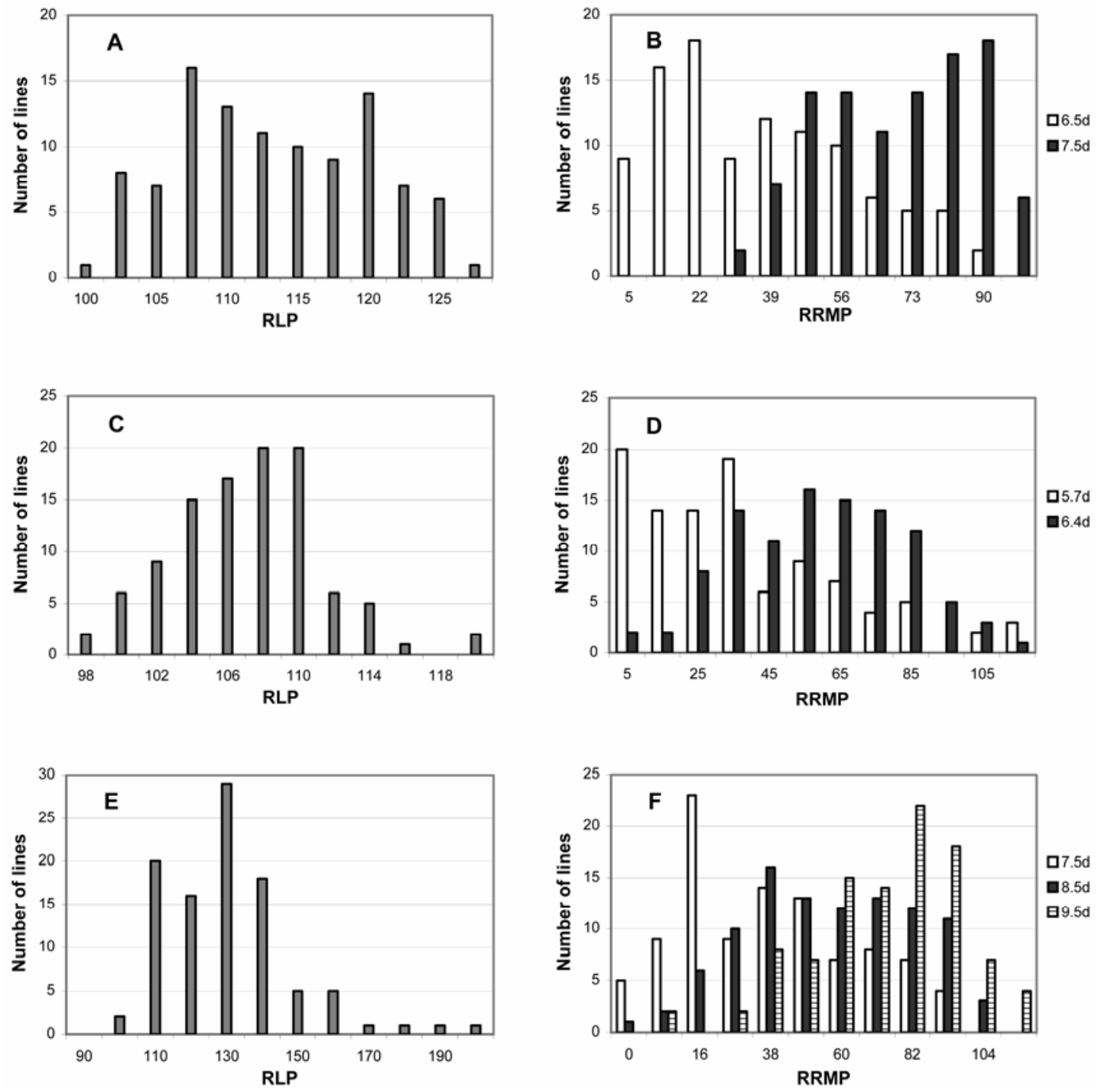

Fig. 2. Frequency distribution of recombinant inbred lines (RILs) derived from a barley cross L94 $\times$ Vada for relative latent period (RLP, left) and relative ratio of mature pustules (RRMP, right) of barley leaf rust in the seedling stage with (A and B) isolate Uppsala (4-replication data) and (C and D) isolate 24 (5-replication data), and (E and $\mathbf{F}$ ) in the adult plant stage with isolate 24 (3-replication data). $\mathrm{d}=$ days postinoculation.

on the RMP in the susceptible control. For example, the optimal time for the first observation of RRMP was when the RMP in susceptible control L94 reached 70 to $90 \%$ at seedling stage, and around 75 to $95 \%$ at adult plant stage. In the present study, this optimal time occurred at around 7.5 dpi with isolate Uppsala and $6.4 \mathrm{dpi}$ with isolate 24 in the seedling stage, and at 8.5 dpi with isolate 24 in the adult plant stage. RMP can be estimated by assuming the final number of mature pustules will equal the number of visible immature pustules plus already mature pustules. Thus, the assessment should be made when about 10 to $30 \%$ of infection sites are still pale flecks in the marked area in the susceptible control. When screening large sets of germplasm, mature and immature pustules should be counted starting when the RMP in the susceptible control has reached 70 to $75 \%$, and the RMP and RRMP could be calculated immediately for each accession. For those accessions with very long latent period, additional immature pustules probably appear after that time, and thus a second counting might be needed to enhance accuracy. By comparing the RRMP of assessed accessions, resistant lines could be easily identified. Of course, the time for assessment depends on the virulence and infecting potential of the race or isolate of the pathogen and the disease development rate. The general development rate depends largely on microclimatic factors such as temperature and on the stage of plant development (2). Therefore, when there is no prior knowledge of the development rate of a pathogen on a set of germplasm in a certain experimental environment, a pre-experiment with the resistant and susceptible controls is recommended to determine what is probably the most suitable time for the first counting. In such a pre-experiment, observations and data collection can be undertaken repeatedly as for LP or AUDPC. Then the suitable time can be determined by making the curve of the ratio of mature pustules in both controls. In general, when the differ-
Table 2. Coefficients of simple correlation $(r)$ between relative ratio of mature pustules (RRMP) at different evaluation times and relative latent period (RLP) of leaf rust in recombinant inbred lines (RILs) of barley

\begin{tabular}{|c|c|c|}
\hline Inoculation stage/isolate & dpi $^{\mathbf{a}}$ & $r^{\mathbf{b}}$ \\
\hline \multirow[t]{4}{*}{ Seedling stage/Uppsala ${ }^{\mathrm{c}}$} & 6.5 & $-0.950^{* *}$ \\
\hline & 7.5 & $-0.983 * *$ \\
\hline & 8.5 & $-0.921 * *$ \\
\hline & 9.5 & $-0.797 * *$ \\
\hline \multirow[t]{3}{*}{ Seedling stage $/ 24^{\mathrm{d}}$} & 5.7 & $-0.792^{* *}$ \\
\hline & 6.4 & $-0.832^{* *}$ \\
\hline & 6.7 & $-0.885^{* *}$ \\
\hline \multirow[t]{7}{*}{ Adult plant stage $/ 24^{\mathrm{e}}$} & 6.5 & $-0.674 * *$ \\
\hline & 7.5 & $-0.772 * *$ \\
\hline & 8.5 & $-0.820 * *$ \\
\hline & 9.5 & $-0.767 * *$ \\
\hline & 10.5 & $-0.726^{* *}$ \\
\hline & 11.5 & $-0.710^{* *}$ \\
\hline & 12.5 & $-0.710^{* *}$ \\
\hline
\end{tabular}

${ }^{a}$ Days postinoculation.

$\mathrm{b} * * P<0.01$.

${ }^{\mathrm{c}}$ Based on four-replication data with isolate Uppsala.

${ }^{\mathrm{d}}$ Based on five-replication data with isolate 24.

e Based on three-replication data with isolate 24. 
Table 3. Comparison of quantitative trait loci (QTLs) detected by restricted multiple-QTL mapping (rMQM) with relative ratio of mature pustules (RRMP) and relative latent period (RLP) for partial resistance to leaf rust in barley

\begin{tabular}{|c|c|c|c|c|c|c|c|}
\hline \multirow[b]{2}{*}{ QTL } & \multirow[b]{2}{*}{ Chromosome } & \multirow{2}{*}{$\begin{array}{c}\text { Map distance } \\
\text { (cM) }\end{array}$} & \multirow[b]{2}{*}{ Peak marker } & \multicolumn{2}{|c|}{ LOD score } & \multicolumn{2}{|c|}{$\operatorname{Exp} \%$} \\
\hline & & & & RLP & RRMPa & RLP & RRMPa \\
\hline \multicolumn{8}{|c|}{ Seedling stage with isolate Uppsala ${ }^{b}$} \\
\hline$R p h q 2$ & $2(2 \mathrm{HL})$ & 185.5 & E38M54-294 & 22.7 & 22.9 & 39.1 & 39.2 \\
\hline Rphq3 & $6(6 \mathrm{HS})$ & 58.2 & E37M33-574 & 9.7 & 9.7 & 13.9 & 13.4 \\
\hline Rphq7 & $7(5 \mathrm{HL})$ & 112.4 & E33M58-306 & 4.0 & 3.5 & 4.2 & 3.6 \\
\hline \multirow{2}{*}{ Rphq8 } & 1 (7HS) & 90.7 & E39M61-372 & 3.2 & 3.4 & 3.5 & 3.6 \\
\hline & & & & & Total & 60.7 & 59.8 \\
\hline \multicolumn{8}{|c|}{ Seedling stage with isolate $24^{\mathrm{c}}$} \\
\hline Rphq2 & $2(2 \mathrm{HL})$ & 185.5 & E38M54-294 & 5.7 & 4.1 & 22.4 & 16.9 \\
\hline Rphq7 & $7(5 \mathrm{HL})$ & 125.0 & E33M55-267 & 5.3 & $2.8^{\mathrm{d}}$ & 16.4 & 9.7 \\
\hline Rphq3 & $6(6 \mathrm{HS})$ & 58.2 & E37M33-574 & 3.9 & 3.4 & 12.3 & 11.8 \\
\hline \multirow[t]{2}{*}{ Rphq3a } & $6(6 \mathrm{HL})$ & 73.3 & E41M40-244 & 3.1 & $2.8^{\mathrm{d}}$ & 10.3 & 10.1 \\
\hline & & & & & Total & 61.4 & 48.5 \\
\hline \multicolumn{8}{|c|}{ Adult plant stage with isolate $24^{\mathrm{e}}$} \\
\hline Rphq3 & $6(6 \mathrm{HS})$ & 58.2 & E37M33-574 & 7.3 & 11.0 & 15.3 & 18.7 \\
\hline Rphq2 & $2(2 \mathrm{HL})$ & 185.5 & E38M54-294 & 5.8 & 6.9 & 11.7 & 12.8 \\
\hline Rphq8 & 1 (7HS) & 90.7 & E39M61-372 & 4.6 & 4.4 & 10.6 & 7.2 \\
\hline Rpgq4 & $7(5 \mathrm{HS})$ & 7.3 & E38M54-247 & 5.2 & 8.9 & 10.2 & 15.1 \\
\hline Rphq10 & 4 (4HS) & 0.0 & E35M54-548 & 3.2 & $2.3^{\mathrm{d}}$ & 6.2 & 3.0 \\
\hline$R p h q 9$ & 1 (7HL) & 129.8 & E33M61-173 & 3.4 & 4.6 & 8.0 & 7.4 \\
\hline \multirow{2}{*}{ Rphq6 } & $2(2 \mathrm{HS})$ & 36.3 & E41M32-83 & & $2.9^{\mathrm{f}}$ & & 5.8 \\
\hline & & & & & Total & 62.0 & 70.0 \\
\hline
\end{tabular}

${ }^{\text {a }}$ RRMP on 7.5 days postinoculation (dpi) with isolate Uppsala and 6.4 dpi with isolate 24 for seedling stage, and 8.5 dpi with isolate 24 for adult plant stage, respectively.

${ }^{\mathrm{b}}$ Based on four-replication data with isolate Uppsala.

${ }^{\mathrm{c}}$ Based on five-replication data with isolate 24 .

d Did not reach threshold value.

e Based on three-replication data with isolate 24.

${ }^{\mathrm{f}}$ Significant for multiple-QTL mapping (MQM) $(\mathrm{LOD}=3.2)$.

Table 4. Averages of relative latent period (RLP) and relative ratio of mature pustules (RRMP) of leaf rust (isolate 1.2.1) in 25 barley lines and cultivars at the seedling stage

\begin{tabular}{lcc}
\hline Line/cultivar & RLP & RRMP \\
\hline K3-59 & 99.3 & 93.9 \\
K2-50 & 100.0 & 95.2 \\
L94 & 100 & 100.0 \\
K2-103 & 100.2 & 100.4 \\
K2-19 & 101.1 & 93.3 \\
K3-109 & 102.2 & 90.6 \\
K2-124 & 102.3 & 91.7 \\
K2-4 & 103.2 & 86.1 \\
Trigo Biasa & 104.3 & 85.6 \\
K2-93 & 104.8 & 80.6 \\
Multan & 105.3 & 80.2 \\
K2-27 & 105.6 & 80.9 \\
Julia & 107.8 & 69.3 \\
Sultan & 109.6 & 66.3 \\
C92 & 109.8 & 62.8 \\
Zephyr & 109.9 & 62.2 \\
Nigrinudum & 110.5 & 59.9 \\
K2-119 & 112.0 & 57.5 \\
$116-5$ & 112.0 & 59.0 \\
FongTien & 112.7 & 60.9 \\
C118 & 114.8 & 45.3 \\
Vada & 126.9 & 20.1 \\
Decorticatum & 128.9 & 24.2 \\
C123 & 133.6 & 10.9 \\
17.5 .16 & 139.0 & 10.4 \\
Mean & 110.23 & 67.48 \\
CV \% & 9.88 & 40.56 \\
$F$ value & $13.56 * *$ & $6.39 * *$ \\
$h_{b}{ }^{2} \%{ }^{c}$ & 96.62 & 84.36 \\
\hline a CV = coefficient of variation. & \\
b $F=$ MS $/$ MS & in a one-way ANOVA. $* * P<$ \\
0.01. & & \\
${ }^{c} h_{b}{ }^{2}=$ broad-sense heritability (genetic variance \\
divided by & phenotypic variance) & based on \\
ANOVA. & & \\
& &
\end{tabular}

ence between susceptible and resistant controls approaches the greatest value, it should be the best moment for the first observation. Therefore, we suppose that this procedure would be suitable for other similar pathosystems, such as slow rusting diseases in wheat (15).

In general, a uniform distribution of inoculum is considered important for assessment of quantitative resistance. Heterogeneous distribution of inoculum over plants may hamper a proper estimation of levels of resistance, since the plants that by chance received more inoculum will seem to be more susceptible. For those measurements based on the quantity of infection on plants, the accuracy of estimated results is largely affected by the homogeneity of the inoculum distribution. For instance, infection frequency (IF) and AUDPC will give inaccurate assessments of resistance if the distribution of inoculum is heterogeneous. An advantage of RRMP, like RLP, as a parameter to assess the level of quantitative resistance in adult plants, is that it does not require a very uniform distribution of inoculum, which is hard to achieve in adult plants. RRMP and RLP depend on the disease development rate and the difference between assessed lines and the susceptible reference in resistance, but not on the number of infection sites on plants. Therefore, these parameters are not affected by fortuitous differences in inoculum densities, provided that there are not too few spores on leaves to produce pustules (too small samples) or too high densities to hamper counting of individual pustules.
Correlation analysis suggested that the average coefficient of correlation among replications for RRMP at optimal times of observation was high (0.68 to 0.78 for experiment I and 0.80 for experiment II), and very close to that for RLP. The estimates of heritability of RRMP at optimal evaluation times were consistently high (84.6 to $94.3 \%$ ) for the two plant growth stages and two isolates, even though the level of host resistance and the size of effects of QTLs in different plant growth stages and to different fungal isolates would vary or differ (16). The effectiveness of RRMP in detecting differences among accessions in partial resistance to leaf rust and identifying the underlying QTLs proved similar to that of RLP at different growth stages of plants and with different isolates. Therefore, RRMP, like RLP, can be expected to produce repeatable results.

In conclusion, RRMP is a simple, rapid, and accurate method to assess quantitative resistance to leaf rust in barley. It is of great use for large-scale monocyclic germplasm screening and evaluation of breeding materials. It also can be effectively employed in fundamental studies, such as identification of QTLs associated with the resistance.

\section{ACKNOWLEDGMENTS}

We thank Wageningen University for granting a fellowship to Guo-Liang Jiang to do this research as a visiting scientist in the framework of the scientific exchange between NAU and WUR. We are also grateful to Xiaoquan Qi for his assistance in QTL mapping. Special thanks are due to Sasha 
Kravchenko, the Department of Crop and Soil Sciences at Michigan State University, for her critical review and comments on the experimental design and analysis.

\section{LITERATURE CITED}

1. Birhman, R. K., and Singh, B. P. 1995. Pathcoefficient analyses and genetic parameters of the components of field resistance of potatoes to late blight. Ann. Appl. Biol. 127:353362 .

2. de Vallavieille-Pope, C., Giosue, S., Munk, L., Newton, A. C., Niks, R. E., Ostergard, H., Pons-Kuhnemann, J., Rossi, V., and Sache, I. 2000. Assessment of epidemiological parameters and their use in epidemiological and forecasting models of cereal airborne diseases. Agronomie 20:715-727.

3. Dorrance, A. E., Inglis, D. A., Helgeson, J. P., and Brown, C. R. 2001. Partial resistance to Phytophthora infestans in four Solanum crosses. Am. J. Potato Res. 78:9-17.

4. Dowkiw, A., Husson, C., Frey, P., Pinon, J., and Bastien, C. 2003. Partial resistance to Melampsora larici-populina leaf rust in hybrid poplars: Genetic variability in inoculated excised leaf disk bioassay and relationship with complete resistance. Phytopathology 93:421-427.

5. Fehr, W. R. 1987. Principles of cultivar development. V. 1. Theory and technique. Macmillan Publishing Company, New York.

6. Iamsupasit, N., Chakraborty, S., Cameron, D. F., and Adkins, S. W. 1993. Components of quantitative resistance to anthracnose (Colletotrichum-gloeosporioides) in tetraploid acces- sions of the pasture legume Stylosantheshamata. Aust. J. Exp. Agric. 33:855-860.

7. Jacobs, T. H., and Parlevliet, J. E. 1993. Durability of disease resistance. Kluwer Academic Publishers, Dordrecht, Netherlands.

8. Jansen, R. C. 1993. Interval mapping of multiple quantitative traits loci. Genetics 135:205211.

9. Jeger, M. J., and Viljanen-Rollinson, S. L. H. 2001. The use of the area under the diseaseprogress curve (AUDPC) to assess quantitative disease resistance in crop cultivars. Theor. Appl. Genet. 102:32-40.

10. Lander, E. S., and Botstein, D. 1989. Mapping Mendelian factors underlying quantitative traits using RFLP linkage maps. Genetics 121:185-199.

11. Newton, A. C. 1989. Genetic adaptation of Erysiphe graminis f. sp. hordei to barley with partial resistance. J. Phytopathol. 126:133-148.

12. Parlevliet, J. E. 1975. Partial resistance of barley to leaf rust, Puccinia hordei. I. Effect of cultivar and development stage on latent period. Euphytica 24:21-27.

13. Parlevliet, J. E. 1995. Durable resistance and how to breed for it. Pages 1-14 in: Breeding for disease resistance with emphasis on durability. D. L. Danial, ed. Wageningen Agricultural University, Wageningen, Netherlands.

14. Parlevliet, J. E., Lindhout, W. H., Van Ommeren, A., and Kuiper, H. J. 1980. Level of partial resistance to leaf rust, Puccinia hordei, in West-European barley and how to select for it. Euphytica 29:1-8.

15. Prabhu, K. V., Luthra, J. K., and Nayar, S. K.
1993. Slow-rusting resistance in wheat (Triticum-aestivum) to leaf rust (Pucciniarecondita) in northern hills of India. Indian $\mathrm{J}$ Agric. Sci. 63:354-357.

16. Qi, X., Jiang, G., Chen, W., Niks, R. E., and Stam, P. 1999. Isolate-specific QTLs for partial resistance to Puccinia hordei in barley. Theor Appl. Genet. 99:877-884.

17. Qi, X., Niks, R. E., Stam, P., and Lindhout, P. 1998. Identification of QTLs for partial resistance to leaf rust (Puccinia hordei) in barley. Theor. Appl. Genet. 96:1205-1215.

18. Qi, X., Stam, P., and Lindhout, P. 1998. Use of locus-specific AFLP markers to construct a high-density molecular map in barley. Theor Appl. Genet. 96:376-384.

19. Robinson, R. A. 1969. Disease resistance terminology. Rev. Appl. Mycol. 48:593-606

20. Sokal, R. R., and Rohlf, F. J. 1981. Biometry: The principles and practice of statistics in biological research. 2nd ed. W. H. Freeman and Company, San Francisco.

21. Van der Plank, J. E. 1963. Plant Disease: Epidemics and Control. Academic Press, New York.

22. Van der Plank, J. E. 1968. Disease Resistance in Plants. Academic Press, New York.

23. Van Ooijen, J. W. 2004. MapQTL® 5, Software for the mapping of quantitative trait loci in experimental populations. Kyazma B. V., Wageningen, Netherlands.

24. Wilson, J. P. 1994. Field and greenhouse evaluations of pearl-millet for partial resistance to Puccinia substriata var. indica. Plant Dis. 78:1202-1205. 